\title{
Magnetic Turbulence and Current Drive during Local Helicity Injection
}

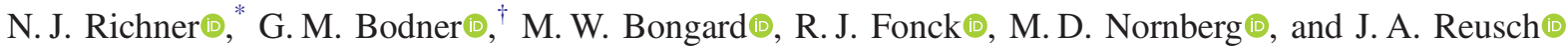 \\ Department of Engineering Physics, University of Wisconsin-Madison, \\ 1500 Engineering Drive, Madison, Wisconsin 53706, USA
}

(Received 2 July 2021; revised 7 December 2021; accepted 20 January 2022; published 7 March 2022)

\begin{abstract}
Magnetic measurements during dc helicity injection tokamak startup indicate Alfvénic turbulence in the injected current streams mediates magnetic relaxation and results in macroscopic plasma current drive. Localization of such activity to the injected current streams, a bias voltage dependence to its onset, and higher-order spectral analysis indicate super-Alfvénic electrons excite instabilities that drive the observed turbulence. Measured fluctuation helicity is consistent with an $\alpha$-dynamo electromotive force driving net current comparable to the macroscopic equilibrium current density. These results imply new constraints for scaling local helicity injection to larger devices.
\end{abstract}

DOI: 10.1103/PhysRevLett.128.105001

The tokamak configuration is a leading concept for the use of magnetic plasma confinement to develop controlled nuclear fusion power [1]. To develop a compact pilot plant concept-which has been identified in recent National Academies and community planning reports as a longterm goal of the U.S. fusion science program [1-3]techniques to initiate and grow the required toroidal plasma current without the use of a central solenoid for magnetic induction are crucially needed [4,5]. In particular, developing an understanding of the underlying physical mechanisms that lead to local current drive is required to confidently extrapolate any such technique to fusion-scale experiments.

The injection of dc magnetic helicity into toroidal systems has been shown to be an effective technique to establish and grow plasma current [5]. While dc helicity injection techniques have been studied in both tokamak and spheromak configurations since the 1980s, the amount of driven current has almost always been described by applying global conservation laws, particularly helicity and energy conservation. However, the local physical mechanisms that convert the injected helicity into largescale current drive are neither well characterized nor understood, limiting the ability to confidently project performance to different configurations or scales.

Magnetic helicity, defined as $K=\int \mathbf{A} \cdot \mathbf{B} d^{3} x$, represents the linkage of poloidal and toroidal magnetic fluxes in a toroidal configuration. Magnetic helicity content is thus directly proportional to the toroidal plasma current $I_{p}$ and

Published by the American Physical Society under the terms of the Creative Commons Attribution 4.0 International license. Further distribution of this work must maintain attribution to the author(s) and the published article's title, journal citation, and DOI. toroidal field $B_{\text {tor }}$, i.e., $K \sim I_{p} B_{\text {tor }}$, for tokamak geometry [6-8]. The injection of magnetic helicity into a plasma, accompanied by relaxation to a helicity-conserved minimum magnetic energy state via electromagnetic instabilities, sustains $I_{p}$ against resistive losses [7,9]. The relevant overall processes involved in such plasma formation and current growth include the following: magnetic turbulence; relaxation and self-organization; heating and transport; and magnetic reconnection $[7,10]$. As such, this problem offers a rich environment for complex nonlinear physics studies towards an important topical application. In addition, studies of current initiation and drive through helicity injection can inform investigations relevant to both laboratory and astrophysical plasmas [11].

This Letter reports the first measurements of local magnetic turbulence excited during a dc helicity injection technique and quantifies its contribution to global current drive in a spherical tokamak configuration. The specific technique employed is called local helicity injection (LHI), wherein relatively small, intense electron current sources inject dc helicity at the edge of a tokamak configuration to both create and grow toroidal plasma current. Experiments show significant broadband magnetic fluctuation activity with properties characteristic of Alfvén wave turbulence. The parametric dependencies of the broadband activity and higher-order spectral analysis implicate beam instabilities in the injected current streams as the driving source of the activity. Estimates of a local fluctuation-driven dynamo current derived from the measured properties of the magnetic activity are comparable to the global current density estimated from equilibrium reconstructions. Altogether, these observations suggest a working hypothesis of a relaxation and current drive mechanism active during LHI that connects the macroscopic behavior of the tokamaklike current channel to local plasma processes. This evolving understanding of these processes will inform 
models for scaling such current drive techniques to fusionscale conditions.

Experimental results from a study of plasmas initiated and sustained by the LHI dc helicity injection scheme [7] are presented here. The high current injectors in the plasma edge region are biased relative to the vacuum vessel to inject dc magnetic helicity $\dot{K}_{\mathrm{dc}}=2 V_{\mathrm{inj}} \psi_{\mathrm{inj}}$, where $V_{\mathrm{inj}}$ is the applied bias and $\psi_{\text {inj }}$ is the magnetic flux through the injector [12]. The injected current streams are unstable to both large-scale MHD fluctuations and small-scale Alfvénic turbulence. These helicity-conserving instabilities and associated magnetic reconnection activity convert the open field line topology into a tokamaklike toroidal state [9].

This work was conducted on the Pegasus Toroidal Experiment at the University of Wisconsin-Madison. Pegasus is an ultralow aspect ratio spherical tokamak $(A \geq 1.1)$ that allows plasma startup and drive via localized dc helicity injectors in the plasma edge region and/or a conventional central Ohmic solenoid [13]. For these studies, LHI-driven deuterium plasmas were formed with a pair of $4 \mathrm{~cm}^{2}$ area washer-stack plasma gun arc sources in the lower divertor region [9], achieving plasma currents $I_{p}$ up to $0.25 \mathrm{MA}$ with total $I_{\text {inj }} \leq 8 \mathrm{kA}$ and $V_{\text {inj }} \sim 1 \mathrm{kV}$.

The extremely low aspect ratio of this experiment provides access to relevant tokamak physics at low toroidal magnetic field strength and modest plasma parameters $\left(T_{e} \sim 100 \mathrm{eV}, n_{e} \sim 10^{19} \mathrm{~m}^{-3}\right)$. Achieving the targeted plasma currents with relatively short pulse lengths ( $20 \mathrm{~ms})$ permits the use of insertable probes [14,15], and thereby provides a unique diagnostic capability of direct, local measurements inside the tokamak plasma edge region. Here, a set of radially translatable magnetic probes [16] were used to study the 3D magnetic activity present during LHI.

As may be expected, LHI-driven plasmas show significantly more magnetic activity compared to those conventionally driven by Ohmic solenoid induction. Figure 1 shows the vertical magnetic field fluctuation power spectral density $\left(d \tilde{b}_{z}^{2} / d f\right)$ in the near-edge region for a representative LHI-driven discharge and a comparable Ohmic plasma with similar $I_{p}=0.15 \mathrm{MA}$, size (major radius $R_{0}=0.35 \mathrm{~m}$, minor radius $a=0.30 \mathrm{~m}$ ), shape (elongation $\kappa=2.17$ ), and $B_{\text {tor }}=0.16 \mathrm{~T}$. LHI fluctuation activity is $\sim 20-30 \mathrm{~dB}$ higher than that of Ohmic drive, with the difference increasing at high frequencies.

Several robust features have been identified in the magnetic spectra over the available range of $|B|, I_{p}$, $V_{\text {inj }}$, and $I_{\text {inj }}$ LHI operational parameters. These are an $n=1$ toroidal mode at $f \approx 10-60 \mathrm{kHz}$ that was previously shown to be consistent with line-tied kinking of the injected current streams during LHI [17]; a peaklike feature at $\approx 500-800 \mathrm{kHz}$ that is associated with instability of the injector plasma arc source itself; and the broadband, turbulent magnetic activity that is the main focus of the present study. During the sustainment phase of LHI,

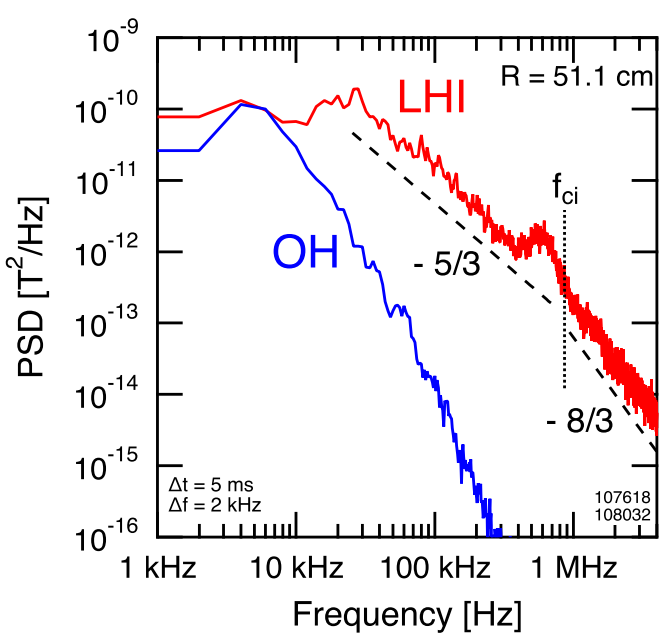

FIG. 1. Magnetic power spectral density within plasma edge for LHI-driven (red) and Ohmic-driven (blue) discharges.

this broadband activity appears to be time stationary $\left[\tilde{b}^{2}(t) \approx\right.$ constant $]$. As such, some unstable mode(s) must be active to inject power into the turbulence and balance the transfer and dissipation of power across the spectrum.

The broadband magnetic fluctuations at $f \sim 100 \mathrm{kHz} \rightarrow$ $4 \mathrm{MHz}$ exhibit power-law behavior $d \tilde{b}^{2} / d f \propto f^{\alpha}$ with spectral indices $\alpha$ consistent with $-5 / 3$ at low frequency and $-8 / 3$ at high frequency (dashed lines in Fig. 1). The location of the spectral break tracks with the local ion cyclotron frequency $f_{c i}$. The measured radial correlation length of the broadband turbulence observed in LHI is $\sim 4 \mathrm{~cm}$, giving $k_{\perp} \rho_{i} \sim 1$. The fluctuations perpendicular to the field are much larger than that parallel to the dominant toroidal field, with $\tilde{b}_{\perp} \gg \tilde{b}_{\|}$. The probability distribution function shape is non-Gaussian, implying temporal intermittency that is associated with small-scale, current-carrying structures $[11,18]$.

The change of magnetic topology during LHI from the coherent, nonaxisymmetric injected current streams to a toroidally averaged large-scale tokamaklike plasma requires magnetic reconnection. Supporting measurements of anomalous ion heating attributed to magnetic reconnection during LHI on PEGASUs showed that the reconnection heating is time correlated with continuous magnetic activity at $f \sim 200-400 \mathrm{kHz}$ and above [19].

The observed spectral properties during LHI are similar to those found in astrophysical systems, particularly for solar wind turbulence [20-22]. In solar wind turbulence, this activity is attributed to MHD Alfvén wave turbulence at large scales $(\alpha=-5 / 3)$ and kinetic Alfvén wave and/or whistler wave turbulence at subion scales $(\alpha \approx-2.7)$ $[21,22]$. Likewise, the magnetic turbulence in astrophysical plasmas is coincident with the presence of magnetic reconnection activity and a significant population of energetic particles. The spatial scales and relative amplitudes of $\tilde{b}_{\perp}$ vs $\tilde{b}_{\|}$also suggest consistency with Alfvénic turbulence [22]. 


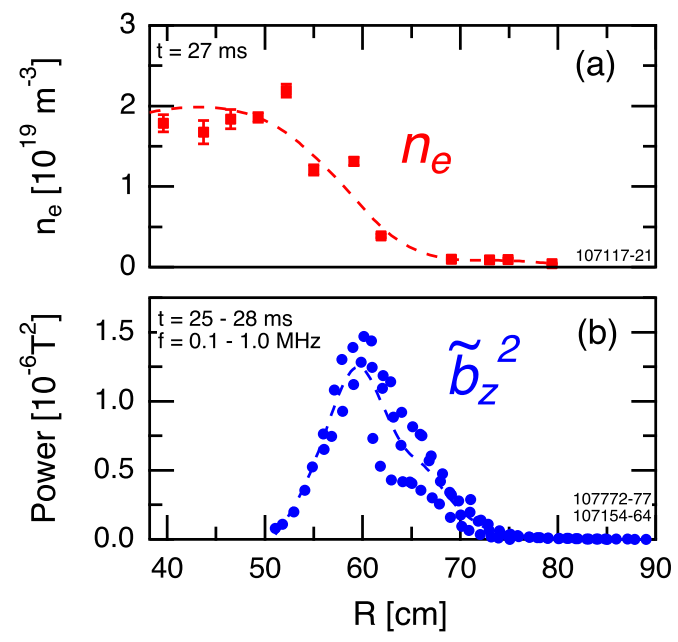

FIG. 2. Radial profiles of (a) plasma electron density and (b) broadband magnetic fluctuation power for $f=0.1-1 \mathrm{MHz}$. Dashed lines are smoothing splines applied to the data. $I_{p}=0.12 \mathrm{MA}, B_{\mathrm{tor}}=80 \mathrm{mT}$.

The presence of Alfvén wave turbulence during LHI provides a candidate for driving the dynamics of the relaxation and current drive. Such magnetic turbulence is generally invoked as a mechanism for magnetic relaxation [10]. Additionally, Alfvénic turbulence exhibits an inverse cascade of magnetic helicity from small to large scales [11,23,24], and coherent beating of fluctuation activity can drive net largescale dc current via the dynamo effect [25-28].

This broadband activity is concentrated in the near-edge region of the tokamaklike plasma. Figure 2 shows radial profiles for the electron density [Fig. 2(a)] as measured with a multipoint Thomson scattering system [29] and the integrated magnetic fluctuation power [Fig. 2(b)] as measured with an insertable probe array [16] from six repeat discharges. The magnetic activity peaks in the edge gradient region and rapidly falls off both further outboard (toward the vacuum vessel wall) as well as further inward (into the confined tokamaklike plasma). Analysis of signals from a distributed Mirnov coil array [17] and more recent measurements with an insertable Hall probe array [30] indicate the injected electron streams are entrained in this same location in the plasma edge and located radially inward of the injector locations projected to the plasma midplane. The fluctuation activity peaking in this same region suggests it is driven in the injected streams themselves. Similar broadband spectra are observed in current stream-only discharges where the tokamaklike state is prevented from forming, further supporting the notion of the activity being driven by the injected electron streams.

An experiment where the injector bias was rapidly shut off and then gradually restored found a strong dependence of the activity on injector voltage. A circuit was installed to transiently null $V_{\text {inj }}$ and linearly ramp to its initial value in $\sim 0.5 \mathrm{~ms}$. The strong injector voltage dependence is shown
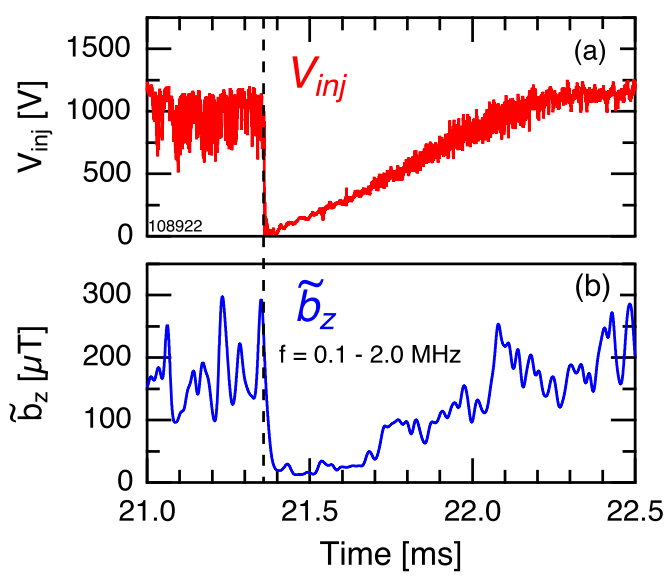

FIG. 3. (a) Injector voltage and (b) amplitude of broadband magnetic activity for $f=0.1-2 \mathrm{MHz}$. Dashed line indicates time of injector shutoff.

in Fig. 3, which plots $V_{\text {inj }}(t)$ [Fig. 3(a)] and the $\tilde{b}_{z}$ amplitude [Fig. 3(b)] for the range of $f=0.1-2 \mathrm{MHz}$ versus time. When $V_{\text {inj }}$ is shut off, the magnetic activity rapidly decays $(\tau \lesssim 15 \mu \mathrm{s})$. As the injector bias is restored, the magnetic activity gradually increases with $V_{\text {inj }}$. This variation with $V_{\text {inj }}$ suggests a dependency on the injected electron beam velocity, which scales as $v_{\text {beam }} \sim \sqrt{V_{\text {inj }}}[19]$.

Such dependencies are common in the instability criteria of beam instabilities [31]. For the nominal operational parameters of LHI on PEGASUS, the expected electron beam velocity at the injectors is estimated as $v_{\text {beam }} \approx$ $1.5 \times 10^{7} \mathrm{~m} / \mathrm{s}$, which corresponds to thermal Mach numbers of 3-6 and Alfvénic Mach numbers of 30-100; the ratio of beam density to background plasma density is $n_{b} / n_{0} \approx 0.1-1$. With the injected electron beams being both superthermal and superalfvenic and having a high relative density, a variety of beam instabilities are predicted to be unstable [32-36].

Altogether, these experimental observations appear to be consistent with a hypothesis of magnetic turbulence being driven in the edge region via streaming instabilities in the injected current streams.

Higher-order power spectral density analysis of the magnetic activity is used to estimate the spectral region of the underlying unstable mode(s). Such techniques enable estimation of the linear growth rate and nonlinear coupling of activity $[37,38]$. The analysis employed here is based on the methods used by Kim et al. [39,40]. For this, the time evolution of each mode $k$ in the system is modeled as the sum of the linear growth or decay $\gamma_{k}$ and the nonlinear power transfer $T_{k}^{k_{1} k_{2}}$ between modes in the system

$$
\frac{\partial P_{k}}{\partial t}=\gamma_{k} P_{k}+\sum T_{k}^{k_{1} k_{2}} .
$$

By approximating this expression as a finite difference and modeling the incremental change of the (complex) power 
spectral density function (PSD) as resulting from a linear and (quadratic) nonlinear transfer function operating on the PSD at an earlier time, expressions for the growth rate and nonlinear power transfer can be calculated.

Such expressions involve higher-order moments of the Fourier transform (such as the bispectrum), and so sufficient ensemble averaging is required to reduce the variance in the computed quantities via this method. The analysis presented here employed data from LHI discharges with $I_{p} \approx 0.1 \mathrm{MA}$ and $B_{\text {tor }}=45 \mathrm{mT}$. Data were selected from a $3 \mathrm{~ms}$ time window in the flattop of the discharge and divided into 150 distinct subwindows for the ensemble averaging. The analysis was performed using a time delay $\tau=1 \mu \mathrm{s}$. To further reduce the variance in the data and to provide an estimate of the standard error, the results for 13 separate probe time histories (spanning a $4 \mathrm{~cm}$ radial extent over three repeat discharges) were averaged together.

Here, frequency is used as a proxy for wave number with $\omega \approx k V$ using the Taylor hypothesis (e.g., from sweeping of quasistationary fluctuations past a sensor) for plasma flow speed $V$. This is justified for fully developed turbulence if $V / v_{a} \gg 2 \pi \delta B_{k} / B$ where $v_{a}$ is the Alfvén speed and $\delta B_{k}$ is the magnetic fluctuation amplitude at wave number $k$ [41]. This condition is satisfied for the broadband fluctuations studied here.

The result of this analysis is shown in Fig. 4, which displays the computed linear growth rate [Fig. 4(a)] and the nonlinear power transfer rate [Fig. 4(b)] as a function of frequency. It indicates modes at intermediate to high frequency $(200-750 \mathrm{kHz})$ are linearly unstable and have
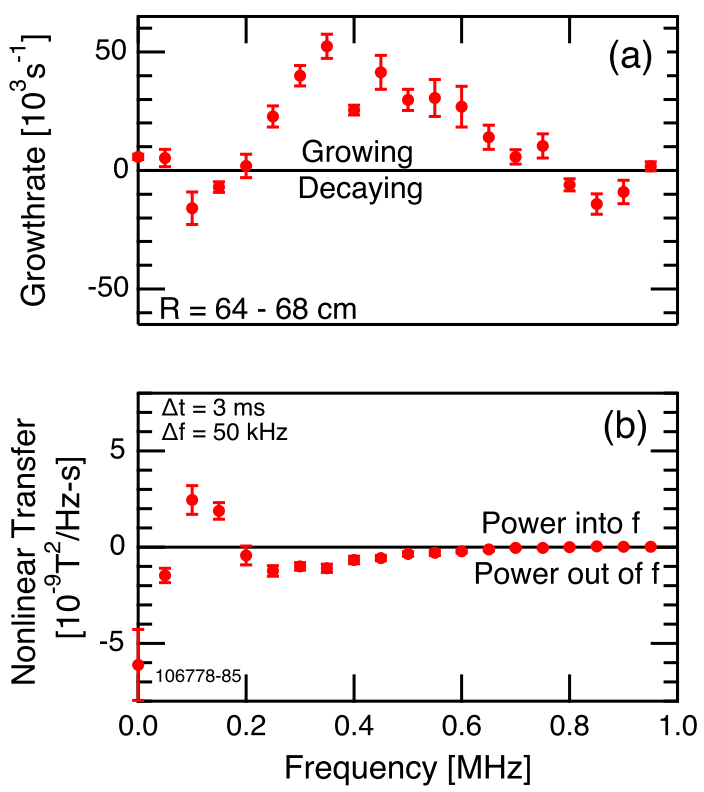

FIG. 4. Estimate of linear growth rate (a) and nonlinear spectral power transfer rate (b) for the magnetic activity present during LHI. $I_{p}=0.10 \mathrm{MA}, B_{\text {tor }}=45 \mathrm{mT}$. net nonlinear power transferred out of the mode (i.e., predominantly transfer power to other frequency bins).

Several instabilities could generate magnetic activity in this range. Specific candidate instabilities currently under consideration are the electron beam Alfvén wave instability [35] and the electron beam kinetic Alfvén wave instability [36]. Such instabilities generate circularly polarized fluctuations with a handedness consistent with the magnetic helicity of the driving electron beam [42]. The circularly polarized fluctuations similarly have net helicity which can drive current $[43,44]$.

Since self-organizing systems can generate and sustain large-scale magnetic fields from small-scale fluctuations via dynamo electromotive forces (EMFs) [26,45], this broadband activity has the potential to be responsible for LHI current drive. To assess this more quantitatively, a crude estimate of time-averaged fluctuation-induced current is calculated using a simple $\alpha$-dynamo model. In the physical picture of the $\alpha$ dynamo, the magnetic field is bent, twisted, and folded to form small-scale Ampèrian loops associated with an electric current [26]. This physical picture is used here to obtain an order-of-magnitude estimate of the toroidal current drive from the measured magnetic activity.

Measurements of the 3D $\tilde{\mathbf{b}}$ properties show strong correlation between radial $\left(\tilde{b}_{R}\right)$ and vertical $\left(\tilde{b}_{Z}\right)$ components that indicate a net right-handed (elliptical) polarization with respect to the applied toroidal magnetic field. The coherence $\gamma_{x y}^{2}$ [46] between these components is $0.2-0.7$. This handedness implies dynamo current drive in the same direction as $I_{p}$. The spatial scale corresponding to this activity is estimated using the frequency-resolved radial coherence length $L_{\text {cor }}$, defined as the $1 / e$ decay length of the coherence function $\gamma_{x y}^{2}(f, R)$ [46,47] in the radial direction. For this, the poloidal spatial size is estimated as equivalent to that of the radial. The averaged field strength of a time-averaged Ampèrian loop is estimated from the mean coherent power between the radial and vertical magnetic field oscillations (crosspower). This use of the ensemble-averaged crosspower accounts for any incoherent activity and/or counteracting effects, and thus estimates the net driven current. Altogether, the estimate of current density using Ampère's law is $J_{\text {tor }} \approx 2 \operatorname{sgn}\left(\theta_{R Z}\right) \sqrt{\left|\left\langle\tilde{b}_{R} \tilde{b}_{Z}\right\rangle\right|} / \mu_{0} L_{\text {cor }}$, where the crossphase between radial and vertical fluctuations $\theta_{R Z}$ is used to differentiate between net cocurrent drive $\left(\theta_{R Z} \sim(\pi / 2)\right)$ and countercurrent drive $\left(\theta_{R Z} \sim-(\pi / 2)\right)$. Figure 5 shows this quantity as a function of frequency calculated with probe measurements within the edge of an LHI-driven discharge. The contribution to toroidal current density decreases with frequency, albeit relatively slowly.

Integrating over frequency, the total estimate for current density is $J_{\text {tor }}=400 \mathrm{kA} / \mathrm{m}^{2}$. This order of magnitude estimate is comparable to both the volume-averaged current density $J_{\text {tor }} \approx 250 \mathrm{kA} / \mathrm{m}^{2}$ as well as the peak current 


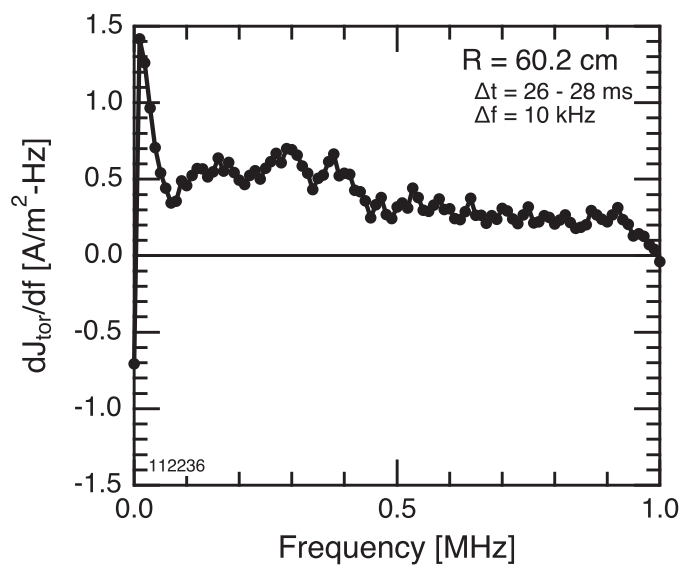

FIG. 5. Estimate of dynamo EMF-driven toroidal current for measured fluctuating magnetic activity versus frequency. $I_{p}=0.12 \mathrm{MA}, B_{\text {tor }}=80 \mathrm{mT}$.

density $J_{\text {tor,max }} \approx 600 \mathrm{kA} / \mathrm{m}^{2}$ inferred from magnetic equilibrium reconstruction of the background plasma. Despite the relative coarseness of this estimate, the general agreement suggests that the broadband activity can account for the observed current drive from LHI through dynamo drives.

Collectively, the observations of the broadband magnetic activity present during LHI motivate a hypothesis for a relaxation and current drive mechanism: (1) beam instabilities in the injected current streams act to drive Alfvénic activity at intermediate frequency ranges; (2) the fluctuations couple nonlinearly, driving a broadband turbulent spectrum; (3) the turbulent cascade of energy and helicity act to relax the plasma system; and (4) resultant dynamo EMFs drive net, large-scale plasma current.

This mechanism is thought to be active in conjunction with a current drive mechanism from discrete, macroscopic stream reconnection events described earlier by O'Bryan et al. [48]. That mechanism and the associated dynamo EMFs are particularly important in the early time dynamics of LHI (especially during the formation of the seed tokamaklike state) [17] but can be suppressed during the sustainment phase of LHI drive [9].

To date, studies of plasma formation and growth via local helicity injection have invoked global concepts such as overall helicity conservation, Taylor relaxation, and tokamak equilibrium constraints to predict plasma performance. The mechanism proposed here of beam-driven magnetic turbulence in the injected current streams that leads to local current drive provides a new, direct link between the global helicity injection, and the small-scale processes that lead to current drive. This physical picture also imposes additional requirements on future application of LHI plasma current startup and growth techniques on larger fusion-grade experiments. That is, the properties of the injected current streams must conform to requirements for excitation of the local magnetic turbulence to support the relaxation process and net current drive.

Data from this publication are publicly available in openly documented, machine-readable formats [49].

The authors thank C. E. Schaefer and C. Pierren for their aid in probe development and operation; B. A. Kujak-Ford, G. R. Winz, and B. T. Lewicki for their technical support; the Pegasus team and students for help in operation; and C. R. Sovinec for valuable discussions. This material is supported by the U.S. Department of Energy, Office of Science, Office of Fusion Energy Sciences, under Awards No. DE-SC0020402 and No. DE-SC0019008. Any opinions, findings, and conclusions or recommendations expressed in this publication are those of the authors and do not necessarily reflect the views of the U.S. Department of Energy.

*Corresponding author.

nrichner@wisc.edu

Present address: General Atomics, P.O. Box 85608, San Diego, California 92186, USA.

Present address: Princeton Plasma Physics Laboratory, 100 Stellarator Road, Princeton, New Jersey 08540, USA.

[1] National Academies of Sciences, Engineering, and Medicine, Bringing Fusion to the U.S. Grid (The National Academies Press, Washington, DC, 2021).

[2] National Academies of Sciences, Engineering, and Medicine, Final Report of the Committee on a Strategic Plan for U.S. Burning Plasma Research (The National Academies Press, Washington, DC, 2019).

[3] S. Baalrud, N. Ferraro, L. Garrison, N. Howard, C. Kuranz, J. Sarff, and W. Solomon, A community plan for fusion energy and discovery plasma sciences, arXiv:2011.04806.

[4] J.E. Menard et al., Fusion nuclear science facilities and pilot plants based on the spherical tokamak, Nucl. Fusion 56, 106023 (2016).

[5] R. Raman and V. F. Shevchenko, Solenoid-free plasma startup in spherical tokamaks, Plasma Phys. Controlled Fusion 56, 103001 (2014).

[6] M. A. Berger and G. B. Field, The topological properties of magnetic helicity, J. Fluid Mech. 147, 133 (1984).

[7] T. H. Jensen and M.S. Chu, Current drive and helicity injection, Phys. Fluids 27, 2881 (1984).

[8] M. A. Berger, Introduction to magnetic helicity, Plasma Phys. Controlled Fusion 41, B167 (1999).

[9] M. W. Bongard, G. M. Bodner, M. G. Burke, R. J. Fonck, J. L. Pachicano, J. M. Perry, C. Pierren, J. A. Reusch, A. T. Rhodes, N. J. Richner, C. Rodriguez Sanchez, C. E. Schaefer, and J.D. Weberski, Advancing local helicity injection for non-solenoidal tokamak startup, Nucl. Fusion 59, 076003 (2019).

[10] J. B. Taylor, Relaxation and magnetic reconnection in plasmas, Rev. Mod. Phys. 58, 741 (1986).

[11] A. Pouquet, D. Rosenberg, J. E. Stawarz, and R. Marino, Helicity dynamics, inverse, and bidirectional cascades in 
fluid and magnetohydrodynamic turbulence: A brief review, Earth Space Science 6, 3 (2019).

[12] D. S. Darrow, M. Ono, C. B. Forest, G. J. Greene, Y. S. Hwang, H. K. Park, R. J. Taylor, P. A. Pribyl, J. D. Evans, K. F. Lai, and J. R. Liberati, Properties of dc helicity injected tokamak plasmas, Phys. Fluids B 2, 1415 (1990).

[13] G. D. Garstka, S. J. Diem, N. W. Eidietis, R. J. Fonck, B. T. Lewicki, G. Taylor, D. J. Battaglia, M. W. Bongard, M. J. Frost, B. A. Kujak-Ford, B. J. Squires, and G. R. Winz, The upgraded Pegasus Toroidal experiment, Nucl. Fusion 46, S603 (2006).

[14] K. E. Thome, M. W. Bongard, J. L. Barr, G. M. Bodner, M. G. Burke, R. J. Fonck, D. M. Kriete, J. M. Perry, and D. J. Schlossberg, High Confinement Mode and Edge Localized Mode Characteristics in a Near-Unity Aspect Ratio Tokamak, Phys. Rev. Lett. 116, 175001 (2016).

[15] M. W. Bongard, R. J. Fonck, C. C. Hegna, A. J. Redd, and D. J. Schlossberg, Measurement of Peeling Mode Edge Current Profile Dynamics, Phys. Rev. Lett. 107, 035003 (2011).

[16] N. J. Richner, M. W. Bongard, R. J. Fonck, J. A. Reusch, and C. E. Schaefer, Radially scanning magnetic probes to study local helicity injection dynamics, Rev. Sci. Instrum. 89, 10J103 (2018).

[17] J. A. Reusch, G. M. Bodner, M. W. Bongard, M. G. Burke, R. J. Fonck, J. L. Pachicano, J. M. Perry, C. Pierren, A. T. Rhodes, N. J. Richner, C. Rodriguez Sanchez, D. J. Schlossberg, and J. D. Weberski, Non-inductively driven tokamak plasmas at near-unity $\beta t$ in the Pegasus Toroidal experiment, Phys. Plasmas 25 (2018).

[18] D. Biskamp, Magnetohydrodynamic Turbulence (Cambridge University Press, Cambridge, England, 2003).

[19] M. G. Burke, J. L. Barr, M. W. Bongard, R. J. Fonck, E. T. Hinson, J. M. Perry, J. A. Reusch, and D. J. Schlossberg, Continuous, edge localized ion heating during nonsolenoidal plasma startup and sustainment in a low aspect ratio tokamak, Nucl. Fusion 57, 076010 (2017).

[20] C. C. Chaston, C. Salem, J. W. Bonnell, C. W. Carlson, R. E. Ergun, R. J. Strangeway, and J. P. McFadden, The Turbulent Alfvénic Aurora, Phys. Rev. Lett. 100, 175003 (2008).

[21] J. P. Eastwood, T. D. Phan, S. D. Bale, and A. Tjulin, Observations of Turbulence Generated by Magnetic Reconnection, Phys. Rev. Lett. 102, 035001 (2009).

[22] O. Alexandrova, C. H. K. Chen, L. Sorriso-Valvo, T. S. Horbury, and S. D. Bale, Solar wind turbulence and the role of ion instabilities, Space Sci. Rev. 178, 101 (2013).

[23] U. Frisch, A. Pouquet, J. Léorat, and A. Mazure, Possibility of an inverse cascade of magnetic helicity in magnetohydrodynamic turbulence, J. Fluid Mech. 68, 769 (1975).

[24] W. C. Müller, S. K. Malapaka, and A. Busse, Inverse cascade of magnetic helicity in magnetohydrodynamic turbulence, Phys. Rev. E 85, 1 (2012).

[25] H. Ji, Turbulent Dynamos, and Magnetic Helicity, Phys. Rev. Lett. 83, 3198 (1999).

[26] H. K. Moffatt, Magnetic Field Generation in Electrically Conducting Fluids (Cambridge University Press, Cambridge, 1978).
[27] R. R. Mett and J. A. Tataronis, Current Drive via Magnetohydrodynamic Helicity Waves, Phys. Rev. Lett. 63, 1380 (1989).

[28] V. S. Chan, R. L. Miller, and T. Ohkawa, Current drive by wave helicity injection, Phys. Fluids B 2, 1441 (1990).

[29] D. J. Schlossberg, G. M. Bodner, M. W. Bongard, R. J. Fonck, J. A. Reusch, and C. Rodriguez Sanchez, A novel, cost-effective, multi-point Thomson scattering system on the Pegasus Toroidal experiment (Invited), Rev. Sci. Instrum. 87, 11E403 (2016).

[30] C. E. Schaefer, G. M. Bodner, M. W. Bongard, R. J. Fonck, J. A. Reusch, and N. J. Richner, Characterization of magnetic structure and activity in local helicity injection, Bull. Am. Phys. Soc. 64, 11 (2019), http://meetings.aps.org/link/ BAPS.2019.DPP.GP10.123.

[31] A. B. Mikhailovskii, Electromagnetic Instabilities in an Inhomogeneous Plasma (Institute of Physics Publishing, Bristol, Philadelphia, New York, 1992).

[32] A. B. Mikhailovskii, Theory of Plasma Instabilities (Consultants Bureau, New York, 1974).

[33] J. Neufeld and H. Wright, Instabilities in a plasma-beam system immersed in a magnetic field, Phys. Rev. 129, 1489 (1963).

[34] R. L. Lysak and W. Lotko, On the kinetic dispersion relation for shear Alfvén waves, J. Geophys. Res. Space Phys. 101, 5085 (1996).

[35] A. Hasegawa, Plasma Instabilities and Nonlinear Effects (Springer, Berlin, Heidelberg, 1975), Vol. 8.

[36] L. Chen, D. J. Wu, G. Q. Zhao, J. F. Tang, and J. Huang, Excitation of kinetic Alfvén waves by fast electron beams, Astrophys. J. 793, 13 (2014).

[37] J. Kim, R. J. Fonck, R. Durst, E. Fernandez, P. Terry, S. Paul, and M. Zarnstorff, Measurements of Nonlinear Energy Transfer in Turbulence in the Tokamak Fusion Test Reactor, Phys. Rev. Lett. 79, 841 (1997).

[38] C. P. Ritz, E. J. Powers, and R. D. Bengtson, Experimental measurement of three-wave coupling and energy cascading, Phys. Fluids B 1, 153 (1989).

[39] J. S. Kim, R. D. Durst, R. J. Fonck, E. Fernandez, A. Ware, and P.W. Terry, Technique for the experimental estimation of nonlinear energy transfer in fully developed turbulence, Phys. Plasmas 3, 3998 (1996).

[40] In the method utilized by Kim et al. to account for nonideal (e.g., noise) power and close the set of equations, the turbulence was assumed to be time stationary. Here, this assumption was relaxed and instead replaced with an assumption that the nonideal power is time stationary.

[41] W. H. Matthaeus and M. L. Goldstein, Measurement of the rugged invariants of magnetohydrodynamic turbulence in the solar wind, J. Geophys. Res. 87, 6011 (1982).

[42] Z. Yoshida and S. M. Mahajan, Decay of Magnetic Helicity Producing Polarized Alfvén Waves, Phys. Rev. Lett. 72, 3989 (1994).

[43] V. S. Chan, R. L. Miller, and T. Ohkawa, Low-frequency current drive and helicity injection, Phys. Fluids B 2, 944 (1990).

[44] R. R. Mett and J. A. Tataronis, Current Drive via Magnetohydrodynamic Helicity Waves, Phys. Rev. Lett. 63, 1380 (1989). 
[45] H. Ji and S. C. Prager, The $\alpha$ dynamo effects in laboratory plasmas, Magnetohydrodynamics 1/2, 191 (2002) [arXiv: astro-ph/0110352].

[46] J. S. Bendat and A. G. Piersol, Engineering Applications of Correlation and Spectral Analysis (John Wiley \& Sons, New York, Chichester, Brisbane, Toronto, Singapore, 1993).

[47] R. D. Durst, R. J. Fonck, G. Cosby, and H. Evensen, Density fluctuation measurements via beam emission spectroscopy (invited), Rev. Sci. Instrum. 63, 4907 (1992).
[48] J. B. O'Bryan and C. R. Sovinec, Simulated flux-rope evolution during non-inductive startup in pegasus, Plasma Phys. Controlled Fusion 56, 064005 (2014).

[49] N. J. Richner, G. M. Bodner, M. W. Bongard, R. J. Fonck, M. D. Nornberg, and J. A. Reusch, Public data set: Magnetic turbulence and current drive during local helicity injection, $10.18138 / 1785967$. 